\title{
Process Alternatives and Scaling Limits for High-density Silicon Tactile Imagers
}

\author{
K. SUZUKI
}

Sensor Research Laboratory, Microelectronics Research Laboratories, NEC Corporation, I120 Shimakuzawa, Sagamihara, Kanagawa 229 (Japan)

K. NAJAFI and K. D. WISE

Center for Integrated Sensors and Circuits, Solid-State Electronics Laboratory, The University of Michigan, Ann Arbor, MI $48109-2122$ (U.S.A.)

\section{Abstract}

In this paper the process complexities and parasitic substrate coupling effects are compared for several different high-density capacitive tactile imagers. The dissolved-wafer process using diffused bulk-silicon row lines and metal-on-glass columns is found to offer the simplest process and fastest response, requiring only five non-critical masks and producing a settling time for the column charge of about $1 \mu \mathrm{s}$. Using this process, a 1024element array with a force range of $1 \mathrm{gm}$ and a spatial resolution of $500 \mu \mathrm{m}$ produces a force resolution equivalent to seven bits. Scaled to a 4096-element array, this same process should produce a force resolution of nearly six bits for the same force range and a spatial resolution of $250 \mu \mathrm{m}$.

\section{Introduction}

Tactile imagers represent one of the most needed devices for precision robotic applications. Although many different approaches have been explored, silicon-based devices remain very attractive due to their potential for high density, high accuracy and low drift. Both piezoresistive and capacitive structures are possible for high-density arrays, each offering a different set of design and performance tradeoffs. For piezoresistive imagers, transducer offset and temperature problems become dominant as element sizes are reduced, whereas for capacitive devices the minimum detectable charge as set by circuit noise limits the resolution of the scaled device [1]. Both piezoresistive and capacitive arrays have been reported for high-density applications $[2,3]$, representing a contrast between deposited (undercut) and dissolved-wafer (bulk) approaches. Piezoresistive polysilicon arrays over sacrificial layers and capacitive structures based on silicon-to-silicon fu- sion bonding are some of the variations that might also be used $[4,5]$. This paper compares five different capacitive structures and three fabrication processes for implementing high-density tactile imaging arrays and examines their performance. Parasitic coupling effects, which limit readout speed, are analyzed. For a 1024-pixel dissolved-wafer capacitive array, results are presented along with the expected scaling behavior of 4096-pixel arrays.

\section{Overall Structure for High-density Capacitive Tactile Imagers}

Figure 1 shows the overall structure for a capacitive tactile imager. An array of forcesensing elements is organized as an $X-Y$ matrix with peripheral circuits for driving voltage on the row lines and detecting the charge induced through the row-column crosspoint capacitors

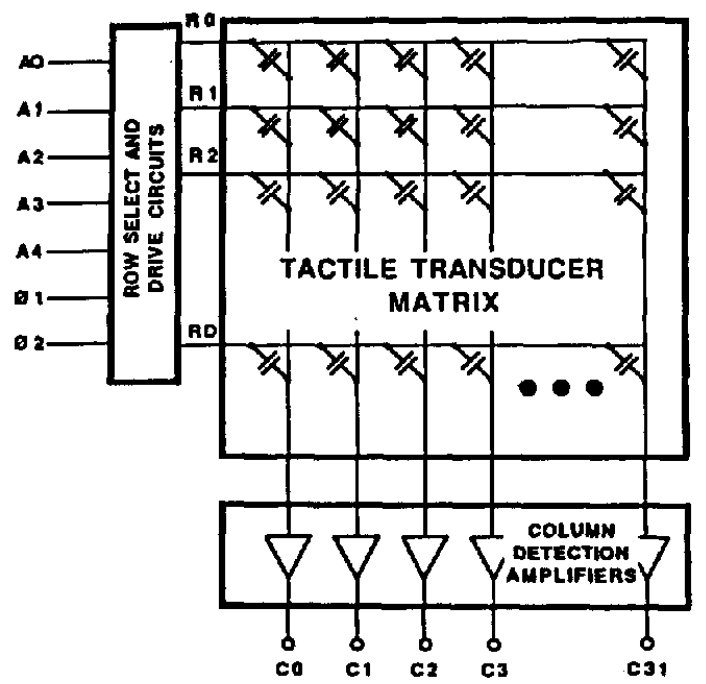

Fig. 1. Overall structure for a high-density capacitive tactile imager.

(C) Elsevier Sequoia/Printed in The Netherlands 
onto the columns. The capacitance of these crosspoints is dependent on the local force on the elements. A switched-capacitor readout scheme is implemented to integrate the charge induced on the column as the selected row line is switched in voltage [6]. A force-independent dummy row line is switched using a voltage opposite to that of the selected row so that the net charge induced on the column is proportional to the difference between the selected crosspoint and the dummy capacitances, allowing the zero-pressure capacitance to be subtracted out. The output voltage of the switched capacitor circuit is independent of the parasitic column capacitance so long as the integrator gain is high. Since there are no active circuits within the array area, this configuration allows a higher element density and considerably simpler process than would be possible in active configurations.

\section{Alternative Structures for the Sensing Element}

Capacitive tactile imagers can be fabricated using a number of technologies, including the bulk-silicon dissolved-wafer process [3], the undercut process using deposited polysilicon films and the silicon-fusion and etch-back approach. Figure 2 shows cross-sectional views of the resulting structures. Although all these structures can be represented by the equivalent circuit shown in Fig. 3(a), the individual circuit parameters are quite different, resulting in different settling times and degrees of crosstalk between the columns. One of the principal parasitic effects limiting the readout speed of the array is the spurious charge induced in the columns due to the bounce in the substrate voltage as the row voltage is switched. This voltage bounce couples additional charge into the column line through the parasitic columnto-substrate capacitance, $C_{\mathrm{Cs}}$. To estimate these coupling effects, the circuit simulation program SPICE PLUS was implemented for $8 \times 8$-element (a)

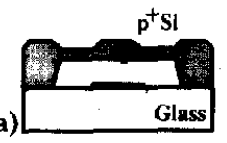

(b)

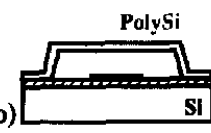

(c)
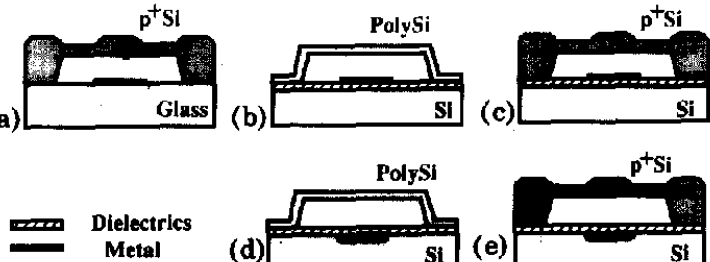
Metal Difrused Laver (e)

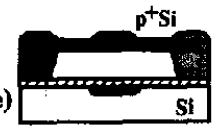

Fig. 2. Different structures for capacitor-based tactile imagers. (a) Dissolved wafer and electrostatic bonding, $\mathbf{p}^{+}$Si-to-metal on glass; (b) poly Si-to-metal on $\mathrm{Si}$; (c) $\mathrm{p}^{+}$Si-to-metal on $\mathrm{Si}$; (d) poly Si-to-diffused layer in $\mathrm{Si}$; (e) $\mathrm{p}^{+} \mathrm{Si-to-diffused} \mathrm{layer} \mathrm{in}$ Si.

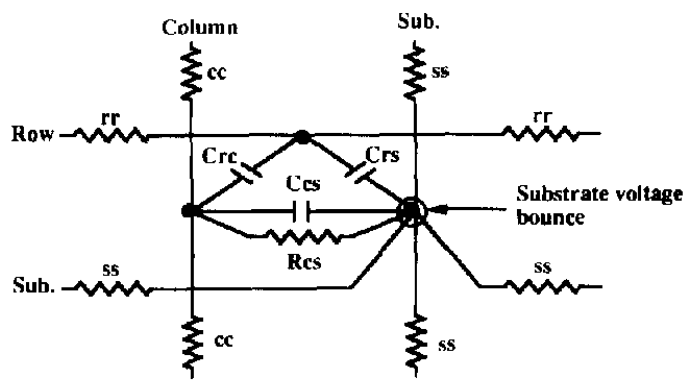

(a)

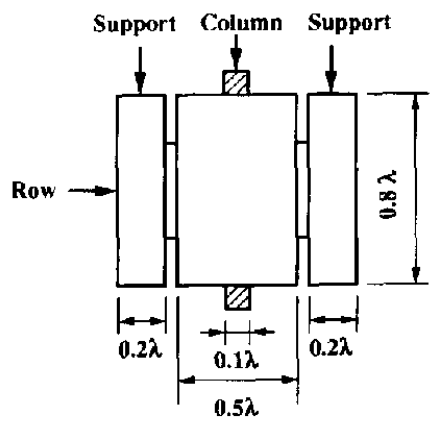

Thickness: $\mathrm{SiO}_{2}: 0.5 \mu \mathrm{m}$; Metal: $0.3 \mu \mathrm{m}$

(b)

Fig. 3. (a) Equivalent circuit for a single element of a tactile imager. $R_{\mathrm{a}}$ is included to aid d.c. convergence. Resistance: $\mathrm{p}^{+}$, $2 \Omega / \square$; diffused layer, $7 \Omega / \square$; metal, $0.03 \Omega / \square$; polysilicon, $70 \Omega / \square$. Capacitance: diffused layer, $0.5 \times 10^{-4} \mathrm{pF} / \mu \mathrm{m}^{2}$ (area); $5 \times 10^{-4} \mathrm{pF} / \mu \mathrm{m}$ (periphery). (b) Dimensions for a single tactile cell used with the parameters in Table $1 . \lambda$ indicates spacing between elements. Separation gap, $2.3 \mu \mathrm{m}$ above silicon surface; thickness, $\mathrm{SiO}_{2} 0.5 \mu \mathrm{m}$, metal $0.3 \mu \mathrm{m}$.

arrays for each of the structures shown in Fig. 2. The equivalent model parameters for each structure are listed in Table 1 , where $\lambda$ is the overall element (pixel) size as shown in Fig. 3(b). Figure 4 shows the transient substrate bounce voltages at a location farthest from the substrate ground for different structures when a $10 \mathrm{~V}$ voltage excursion is applied into the row line in $30 \mathrm{~ns}$. For the structures with diffused silicon column lines (Figs. 2(d) and (e)), the substrate response is relatively slow due to the substrate resistance and the large parasitic row-substrate capacitance. The responses for the metal-on-dielectric column structures in (b) and (c) are more rapid. The substrate bounce voltage decreases more rapidly for the $\mathrm{p}^{+}$ silicon row line and metal column in Fig. 2(c) than for the poly row line in (b) due to the lower resistance of the diffused bulk row line. Since the charge induced onto the column lines is the product of the substrate bounce voltage and the distributed column-substrate capacitance, and since this capacitance is large for all of these structures, the 
TABLE 1. Parameters in the equivalent circuit, shown in Fig. 3(a), for the different capacitor-based tactile imagers of Fig. 2. Substrate resistance values are selected as low as possible to suppress the substrate coupling effects

\begin{tabular}{llllll}
\hline & $(a)$ & $(b)$ & $(c)$ & $(d)$ & $(e)$ \\
\hline$C_{\mathrm{rc}}[\mathrm{pF}]$ & $1.8 \times 10^{-6} \lambda^{2}$ & $1.8 \times 10^{-6} \lambda^{2}$ & $1.8 \times 10^{-6} \lambda^{2}$ & $1.5 \times 10^{-6} \lambda^{2}$ & $1.5 \times 10^{-6} \lambda^{2}$ \\
$\left.C_{\mathrm{rs}} \mathrm{pF}\right]$ & 0 & $2.3 \times 10^{-5} \lambda^{2}$ & $2.3 \times 10^{-5} \lambda^{2}$ & $2.3 \times 10^{-5} \lambda^{2}$ & $2.3 \times 10^{-5} \lambda^{2}$ \\
$C_{\mathrm{cs}}[\mathrm{pF}]$ & 0 & $2.8 \times 10^{-5} \lambda^{2}$ & $2.8 \times 10^{-5} \lambda^{2}$ & $2 \times 10^{-5} \lambda^{2}$ & $2 \times 10^{-5} \lambda^{2}$ \\
$\operatorname{rr}[\Omega]$ & 2 & 70 & 2 & $+1.3 \times 10^{-3} \lambda$ & $+1.3 \times 10^{-3} \lambda$ \\
$c c[\Omega]$ & 0.06 & 0.06 & 0.06 & 14 & 2 \\
$s s[\Omega]$ & $10^{12}$ & 10 & 10 & 100 & 14 \\
$R_{\mathrm{cs}}[\Omega]$ & $\left(10^{12}\right)$ & $\left(10^{12}\right)$ & $\left(10^{12}\right)$ & $\left(10^{12}\right)$ & $\left(10^{12}\right)$ \\
\hline
\end{tabular}

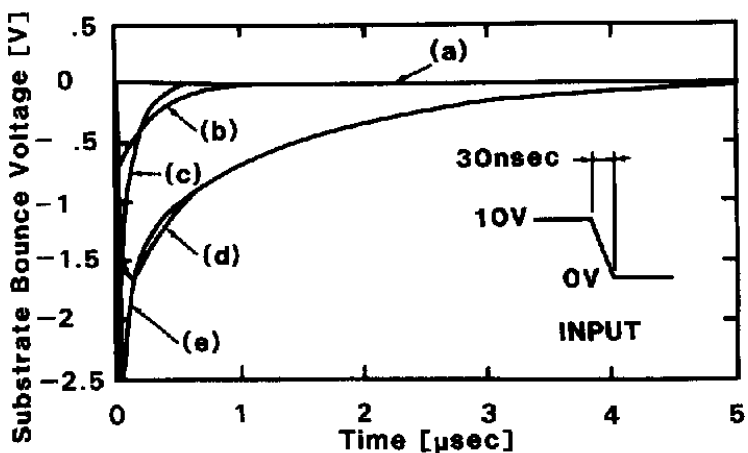

Fig. 4. Substrate voltage bounce for an $8 \times 8$-element array $(\lambda=2 \mathrm{~mm})$ using the different structures shown in Fig. 3.

induced charge is still very significant at $1 \mu \mathrm{s}$. Even for the structure in Fig. 2(c), the substrateinduced noise charge resulting from substrate bounce is still approximately $1 \mathrm{pC}$ for an eightpixel column and $\lambda=2 \mathrm{~mm}$. It is important that this charge does not overload the integration amplifiers or readout integrity will be lost. As the number of elements increases, a longer time is required for these coupling effects to subside due to the increase in the parasitic capacitance as compared to the transducer capacitance. For the structure in Fig. $2(a)$, the substrate voltage bounce is negligible due to the almost perfect isolation of the glass substrate, resulting in a very fast response.

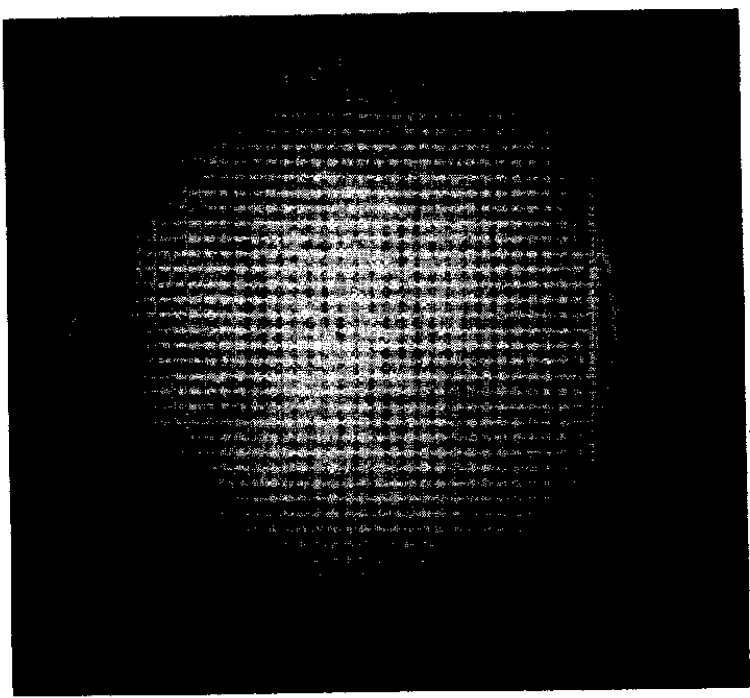

Fig. 5. A completed $32 \times 32$-element tactile imager shown on a U.S. Lincoln cent [3].

Table 2 compares these capacitive structures in terms of their process complexity and design flexibility. The dissolved-wafer process offers the simplest process, has very high yield and can provide the resolution and sensitivity required for a wide variety of applications. A 1024-element imager based on this process has been fabricated [3] and is shown in Fig. 5. The glass substrate measures $2.2 \mathrm{~cm} \times 2.0 \mathrm{~cm}$ and is shown on a Lincoln penny.

TABLE 2. Comparison of dissolved wafer, undercut polysilicon and silicon-silicon bonding techniques for the fabrication of tactile imagers

\begin{tabular}{llll}
\hline Item & Dissolved wafer & Undercut polysilicon & Silicon-silicon fusion \\
\hline Number of masks & $3(2) / 4(2)$ & $4 / 5$ for $(b), 5 / 6$ for $(d)$ & $3(2) / 4(2)$ for $(c), 3(3) / 4(3)$ for $(e)$ \\
Process complexity & Simple & Moderate/high & Moderate \\
Process sensitivity & Low & High & Moderate \\
Uniformity & Good & Good & Moderate/good \\
Yield & High & High/moderate & High/moderate \\
Sensing type & Capacitive & Capacitive/resistive & Capacitive/resistive \\
Sensitivity & High & High & High \\
Parasitics & Low & Moderate/high & Moderate/high \\
Circuit compatibility & No (Hybrid) & Yes & Yes \\
Upward scalability & Good & Moderate/low & Good \\
\hline
\end{tabular}


The imager utilizes a doubly-supported bridge structure that offers flexibility in setting the force sensitivity and the operating force range and lends itself easily to high-density arrays. The use of hybrid flip-chip circuits on the glass for multiplexed drive and readout appears to be an attractive alternative to monolithic circuits for this application due to the very large array size and the need for a simple array process.

\section{Packaging Efiects and Scaling Behavior}

In use, the tactile imager of Fig. 2(a) is coated with a thin metallized pad. This pad electrically insulates the row lines from the external world and seals the areas under the transducing bridges from particulates. Parasitic coupling might also occur through this pad; however, simulations for pad thicknesses from $1 \mu \mathrm{m}$ to $10 \mu \mathrm{m}$ have shown the voltage bounce and induced column charge due to the presence of the pad to be negligible.

Since the crosspoint capacitance decreases rapidly with the dimensions, scaling the array to higher pixel densities to achieve higher spatial resolution results in decreased force resolution. The scaling limit for a given desired force range is thus determined by the minimum detectable charge, as set by the various readout noise sources (thermal noise, noise due to charge leakage, temperature drift, and reset noise charge [6]). For the $32 \times 32$-element array shown in Fig. 5, the minimum detectable charge is about $19 \mathrm{fC}$. Table 3 shows the scaling behavior of this capacitive tactile imager, based on the doubly-supported bridge

TABLE 3. Scaling behavior for a high-density tactile imager fabricated by the dissolved wafer technique

\begin{tabular}{|c|c|c|c|}
\hline Element number & $16 \times 16$ & $32 \times 32$ & $64 \times 64$ \\
\hline $\begin{array}{l}\text { Cell spacing } \\
\text { [mm] }\end{array}$ & 1 & 0.5 & 0.25 \\
\hline $\begin{array}{l}\text { Thin beam } \\
{\left[\mu \mathrm{m}^{3}\right]}\end{array}$ & $600 \times 268 \times 4.8$ & $300 \times 134 \times 3$ & $150 \times 67 \times 1.9$ \\
\hline $\begin{array}{l}\text { Thick genter plate } \\
{\left[\mathrm{mm}^{3}\right]}\end{array}$ & $826 \times 506 \times 12$ & $413 \times 253 \times 12$ & $206 \times 126 \times 12$ \\
\hline $\begin{array}{l}\text { Plate separation } \\
\text { [um] }\end{array}$ & 2 & 2 & 2 \\
\hline $\begin{array}{l}\text { Zero force capacitance } \\
\text { [fF] }\end{array}$ & 1248 & 312 & 78 \\
\hline \multicolumn{4}{|l|}{ Force range } \\
\hline$[g F]$ & 1 & 1 & 1 \\
\hline $\begin{array}{l}\text { Terminal sensitivity } \\
\text { [fF/gf] }\end{array}$ & 1248 & 312 & 78 \\
\hline $\begin{array}{l}\text { Min. delectable charge } \\
\text { [fC] }\end{array}$ & 33 & 19 & 15 \\
\hline $\begin{array}{l}\text { Resolution } \\
\text { [bit] }\end{array}$ & 9 & 7 & 6 \\
\hline
\end{tabular}

structure [3]. A full-scale force range of $1 \mathrm{gm}$ is assumed, at which point the zero force capacitance is designed to have doubled. Even for a $64 \times 64$-element array (4096 pixels), a force resolution of nearly 6 bits is achieved with a spatial resolution of $250 \mu \mathrm{m}$.

\section{Conclusions}

Several structures for implementing silicon capacitive high-density tactile imagers have been examined. The dissolved-wafer process using diffused bulk silicon row lines and metal-on-glass columns offers the fastest response, simplest process and greatest design flexibility. The element response to force is highly independent of the overlying pad, which acts only to transmit force to the transducing bridge structures and seal them from the external world. While the present 1024element arrays have been implemented using offchip discrete electronics, the use of hybrid circuitry on the glass should minimize interconnects and simplify imager fabrication for a wide variety of important high-precision applications.

\section{Acknowledgements}

The devices reported here were designed, fabricated and characterized in the Center for Integrated Sensors and Circuits at The University of Michigan. The simulations and structural comparisons were accomplished in the Microelectronics Research Laboratories of NEC Corporation.

\section{References}

1 H. L. Chau and K. D. Wise, Scaling limits in batch-fabricated silicon pressure sensors, IEEE Trans. Electron Devices, $E D-34(1987) 850-858$.

2 S. Sugiyama, K. Kawahata, A. Abe, H. Funabashi and I. Igarashi, High-resolution silicon pressure imager with CMOS processing circuits, Proc. $4 \mathrm{th}$ Int. Conf. Solid-State Sensors and Actuators (Transducers '87), Tokyo, Japan, June 2-5, 1987, pp. 444-447.

3 K. Suzuki, K. Najafi and K. D. Wise, A 1024-element high-performance silicon tactile imager, Tech. Digest, IEEE '88 IEDM, Dec. 1988, pp. 674-677.

4 R. Howe and R. Muller, Resonant-microbridge vapor sensor, IEEE Trans. Electron Devices, ED-33 (1986) 499-506.

5 K. Petersen, P. Barth, J. Poydock, J. Brown, J. Mallon and J. Bryzek, Silicon fusion bonding for pressure sensors, Tech. Digest, IEEE Solid-State Sensor and Actuator Workshop, Hilton Head Island, SC, U.S.A., June 1988, pp. 143-147.

$6 \mathrm{~K}$. Chun and K. D. Wise, A high-performance silicon tactile imager based on a capacitive cell, IEEE Trans. Electron Devices, ED-32 (1985) 1196-1201. 